\title{
Dynamical overlap fermions: simulation and physics results
}

\author{
Stefan Schaefer* \\ University of Colorado \\ E-mail: Schaeferepizero.colorado.edu \\ Thomas DeGrand \\ University of Colorado \\ E-mail: degrandepizero.colorado.edu
}

\begin{abstract}
We summarize our recent investigations of lattice QCD with dynamical overlap fermions. We sketch algorithmic issues and our approach to solving them. We show our measurement of the topological susceptibility. We describe a computation of the chiral condensate using an analysis of the distribution of eigenmodes of the Dirac operator and Random Matrix Theory.
\end{abstract}

XXIIIrd International Symposium on Lattice Field Theory

25-30 July 2005

Trinity College, Dublin, Ireland

\footnotetext{
*Speaker.
} 


\section{Introduction.}

More than twenty years' experience of the lattice gauge community has taught us that it is always a good thing to have a bare action which respects symmetries, because then no fine tunings are required to preserve the symmetries at distances much greater than the cutoff scale. For this reason, essentially all lattice simulations of gauge theories perform simulations with gauge invariant lattice actions, and there is never any discussion about trading a small violation of gauge invariance in the simulation for larger volume or an apparently more efficient simulation algorithm. People have, however, always been willing to sacrifice chiral symmetry in their choice of lattice discretization. This seems somehow asymmetric: Why not perform simulations with lattice actions which preserve exact $S U\left(N_{f}\right) \times S U\left(N_{f}\right)$ chiral symmetry?

The advantages of this approach are obvious: One does not have to separate the physical explicit chiral symmetry breaking from a nonzero quark mass from the unphysical chiral symmetry breaking induced by lattice artifacts. The flavor content of the theory being simulated is unambiguous. The index theorem is theoretically clean. The topological charge can be measured to be exactly what the dynamical fermions see during the simulation, not something which is determined by some post-processing procedure. And because the action preserves symmetries, correlation functions obey Ward identities which considerably simplify their theoretical analysis. For example, one does not have to spend any time measuring (and trying to remove) lattice-artifact additive mass renormalization or operator mixing.

The way to do this is well known: use a lattice action which encodes the Ginsparg-Wilson[1] relation, an overlap[2, 3] action. This article is a summary of our experiences with simulations of two flavors of dynamical overlap fermions, using a version of the algorithm of Fodor, et al[ 4 ]. It is a condensation of our two recent papers, Refs. [5, 6], plus a little newer material.

\section{Algorithmic Issues}

Simulations with dynamical overlap have (at least) two problems:

- They run so slowly

- Changing topology is hard

Our method of attack for the first problem is to replace the usual link variable gauge connection by a fat link. It has been known since at least 1998 [7, 8, 2, 10, that fat links improve the chiral properties of non chiral fermion actions (and the flavor symmetry properties of staggered fermions). The bottleneck has always been to find a smearing method which can be used in a molecular dynamics update, where the evaluation of the force requires a fat link which is differentiable with respect to its component thin links. Formulations like the Asqtad link solve this problem by "following the paths," but this does not give as much improvement as one would like. Our solution was provided by Peardon and Morningstar[1]] with the "stout link" (invented in a Dublin public house): a multilevel blocking which is fully differentiable. In our runs, it pushes the thin link plaquette $\operatorname{Tr} U_{p} \sim 1.7$ up to about 2.8 (with two levels of smearing with $\rho=0.15$ ). The number of Dirac operator matrix times vector multiplies per trajectory is reduced by about an 
order of magnitude compared to simulations with a thin link gauge connection. The physics reason for this speedup is that fattening reduces the number of small eigenmodes of the kernel operator, improving its conditioning number.

With a stout link, the fermions decouple from the UV fluctuations of the gauge field, and the mean size of the fermion force is reduced to about an order of magnitude smaller than the gauge force. This makes a multiple time scale integration algorithm very attractive. We run with the Sexton-Weingarten[12] form of this updating, taking the integration time step for the gauge fields to be $1 / 12$ of that for the fermions.

The square of the Hermitian overlap operator projected on one chiral sector is given by

$$
H_{\sigma}^{2}(m)=2\left(R_{0}^{2}-\frac{m^{2}}{4}\right) P_{\sigma}\left[1+\sigma \sum_{i} \varepsilon\left(\lambda_{i}\right)\left|\lambda_{i}\right\rangle\left\langle\lambda_{i}\right|\right] P_{\sigma}+m^{2}
$$

with $R_{0}$ the radius of the Ginsparg-Wilson circle, $P_{\sigma}=\frac{1}{2}\left(1+\sigma \gamma_{5}\right)$ the projector on chirality $\sigma$ and $h\left(-R_{0}\right)$ the Hermitian kernel operator. The sign function $\varepsilon\left(h\left(-R_{0}\right)\right)$ is here given in its spectral representation.

Because of the sign function in its definition, the effective action of the overlap operator has a discontinuity. It occurs when one eigenvalue of the kernel $h\left(-R_{0}\right)$ changes sign during the molecular dynamics evolution. These are the surfaces in the space of the gauge fields on which the topology as defined by the index theorem changes by one unit. Ref. [ [\$ gives a prescription of how to account for this discontinuity in the HMC algorithm. One essentially measures the height $\Delta S$ of the step in the action (the potential of our Hamiltonian equations of motion) and if the momentum perpendicular to the surface is large enough one reduces it as one would do in classical mechanics. We will call this a "refraction" in the following. If the perpendicular momentum is too small, we flip it, and thus reflect the trajectory. With $N$ the vector normal to the surface momenta $\pi$ are thus updated by

$$
\Delta \pi= \begin{cases}-N\langle N \mid \pi\rangle+N \operatorname{sign}\langle N \mid \pi\rangle \sqrt{\langle N \mid \pi\rangle^{2}-2 \Delta S_{f}} & \text { if }\langle N \mid \pi\rangle^{2}>2 \Delta S_{f} \\ -2 N\langle N \mid \pi\rangle & \text { if }\langle N \mid \pi\rangle^{2} \leq 2 \Delta S_{f}\end{cases}
$$

The discontinuity $\Delta S$ of the effective action is caused by one eigenvalue changing sign, thus making the replacement

$$
H_{\sigma}^{2}(m) \longrightarrow H_{\sigma}^{2} \pm\left(4 R_{0}^{2}-m^{2}\right) P_{\sigma}\left|\lambda_{0}\right\rangle\left\langle\lambda_{0}\right| P_{\sigma}
$$

with $\left|\lambda_{0}\right\rangle$ the zero mode. The corresponding step in the effective action can be evaluated using the Sherman-Morrison formula [13]

$$
\Delta\left[\left\langle\phi\left|P_{\sigma} \frac{1}{H_{\sigma}(m)^{2}} P_{\sigma}\right| \phi\right\rangle\right]=\mp \frac{\left(4 R_{0}^{2}-m^{2}\right)}{1 \pm\left(4 R_{0}^{2}-m^{2}\right)\left\langle\lambda_{0}\left|P_{\sigma} H_{\sigma}^{-2}(m) P_{\sigma}\right| \lambda_{0}\right\rangle}\left|\left\langle\phi\left|P_{\sigma} \frac{1}{H_{\sigma}(m)^{2}} P_{\sigma}\right| \lambda_{0}\right\rangle\right|^{2} .
$$

Interestingly, for the overlap not only can one compute the step in the effective action, but one can also give a closed form expression for the change in the fermionic determinant due to the change in topology:

$$
\frac{\operatorname{det} \tilde{H}_{\sigma}^{2}(m)}{\operatorname{det} H_{\sigma}^{2}(m)}=1 \pm\left(4 R_{0}^{2}-m^{2}\right)\left\langle\lambda_{0}\left|P_{\sigma} \frac{1}{H_{\sigma}^{2}(m)} P_{\sigma}\right| \lambda_{0}\right\rangle
$$


In the actual simulation one faces the problem that the trajectories reflect most of the time off the zero eigenvalue surface and one never changes topology. The reason is that the change in the determinant from the starting configuration to the 'current' configuration in the MD evolution is only approximated well as long as the Dirac operator is similar to the starting one. The fluctuations are small as long as one has not changed topology. However, this is definitely not the case for the operator in a different topological sector. Since $\exp \left(-\phi^{+} 1 / H^{2} \phi\right)$ averages to the change in the determinant from the starting configuration to the end, large fluctuations mean that most of the time $\exp \left(-\phi^{+} 1 / H^{2} \phi\right) \approx 0$ and the effective action is thus large, whereas only a few times do we get small effective actions. These two observations combine to give large $\Delta S$ most of the time, and thus a large auto-correlation time in the topological charge.

To reduce fluctuations, we used the method proposed in Refs. [14, 15], which consists of rewriting the fermion determinant as as

$$
\operatorname{det} H^{2}(m)=\operatorname{det} H^{2}\left(m_{N_{p}}\right) \prod_{i=1}^{N_{p}-1} \operatorname{det} \frac{H^{2}\left(m_{i}\right)}{H^{2}\left(m_{i+1}\right)}
$$

with $m_{1}=m$ and $m_{i}<m_{i+1}$ with suitably chosen larger masses. In this method, only determinant ratios are evaluated using pseudo-fermions for the light quark masses. The change in the spectra while changing topological sector of the ratio $H(m) / H\left(m^{\prime}\right)$ is expected to be less dramatic than the change of the spectrum of $H(m)$. Only the determinant of $H\left(m_{N}\right)$ is evaluated directly. However, for a large mass $m_{N}$ the spectrum of $H^{2}$ is confined to a smaller region between $m_{N}^{2}$ and $4 R_{0}^{2}$ and the change in the spectrum therefore less drastic than for a smaller mass. One or two extra pseudofermion fields $\left(N_{p}=2,3\right.$ in Eq. 2.6) help some, but do not solve the problem.

To quantify our difficulty, we compare in Fig. 1 the discontinuity of the effective action with the physical step from the fermion determinant. We subtracted the relevant quantity from the normal component of the momentum so that positive values correspond to reflections whereas the topology changes for negative values. We observe that the physical discontinuity would allow for significantly more changes in topology than the step in the effective action does.

The low correlation between the estimator and the physical step height Eq. (2.5) shows up in the large auto-correlation time of the topological charge. Even though part of it is physics - lighter quarks make it harder to get from $v=0$ to $v= \pm 1$ - the height of the step grows with $1 / \mathrm{m}^{2}$ instead of the expected determinant ratio, $\log \mathrm{m}$. Since the normal component of the momentum is roughly independent of the quark mass, it becomes more and more difficult to change topology. Indeed, Fig. 目 shows that the mean time between topological changes varies inversely with the square of the quark mass. The large auto-correlation time for the topology is a phenomenon that is also known with other fermions, e.g. improved staggered quarks. To the extent that these formulations know about topology, the step in the fermion action for the overlap might be replaced for them by a steep region which approximates the step. The result is the same: if the approximation of the determinant is bad, the step is overestimated most of the time and one does not change topology.

\section{The Topological Susceptibility}

In our second paper we made rough calculations of the topological susceptibility and chiral condensate using eigenmodes of the Dirac operator. We made simulations on $8^{4}$ lattices, at a lattice 
spacing of about $a \sim 0.16 \mathrm{fm}$, with three quark masses, $a m_{q}=0.03,0.05$, and 0.1 . These lattices are really too small for physics, but they illustrate the useful features of a calculation with overlap fermions.

We determined the string tension from the heavy quark potential and the Sommer parameter, from Wilson loops of temporal extent $t=2$ and 3. The two measurements are not consistent, but we performed tests on $8^{4}$ and $12^{4}$ quenched lattices which showed that the $t=3$ potentials were consistent with ones from further separations. So we used their fit values. The lattice spacing varies by about ten percent as we change the quark mass.

One picture, Fig. 3. illustrates our measurement of the topological susceptibility $\chi$. We take our measurements of $r_{0} / a$ and the topological charge time history to compute $\chi r_{0}^{4}$. We have computed the lattice-to- $\overline{M S}$ matching factor in perturbation theory and use it to convert the quark masses to their $\mu=2 \mathrm{GeV} \overline{M S}$ values. Dürr[16] has presented a phenomenological interpolating formula for the mass dependence of the topological susceptibility, in terms of the condensate $\Sigma$ and quenched topological susceptibility $\chi_{q}$,

$$
\frac{1}{\chi}=\frac{N_{f}}{m_{q} \Sigma}+\frac{1}{\chi_{q}}
$$

Taking $\Sigma$ from our RMT analysis in the next section $\left(r_{0}{ }^{3} \Sigma \sim 0.43\right)$ produces the curve shown in the figure.

Most published measurements of the topological susceptibility present them as a function of the pseudoscalar mass. Since we don't have spectroscopy, we can't do that. We can, however, use the Dürr curve as a fiducial, since most published measurements of the topological susceptibility present it, too. Our data (as well as that of Ref. [17]) lies below the Dürr curve. Most measurements with nonchiral actions lie above it. (See, for example the figures in Ref. [18] or [19]). Since our quenched results give a value typical of simulations on larger lattices, $\left.\chi \sim(190 \mathrm{MeV})^{4}\right)$, we don't think we are seeing a finite volume effect.
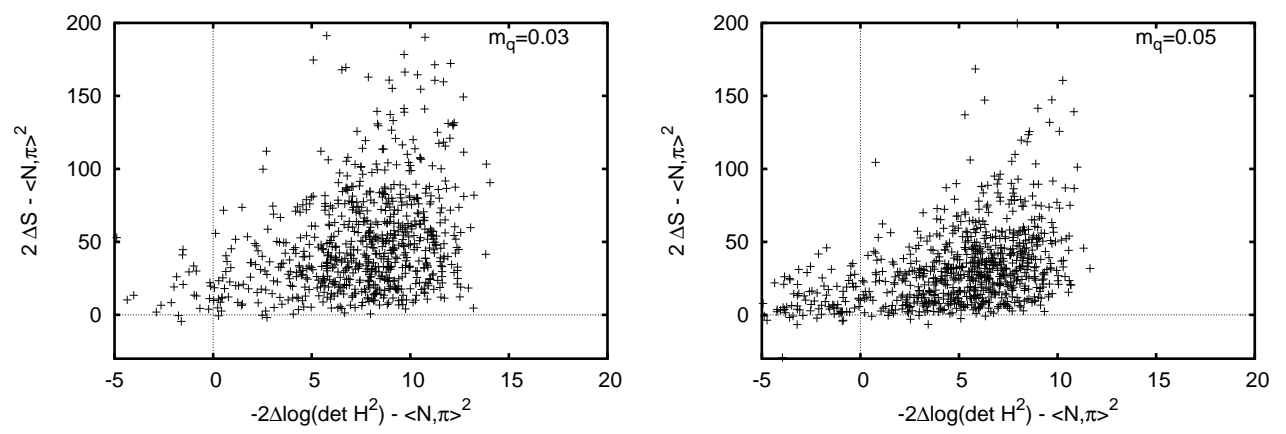

Figure 1: The stochastic estimate of the height of the step compared to the actual change in the logarithm of the determinant from a subset of our ensemble. We subtracted the normal component of the momentum squared (which is typically less than 10) such that negative values mean refraction and positive ones reflection. For mass $m_{q}=0.03$ on the left we have a number of events in the upper left quadrant that would have tunneled with the exact change of the determinant and only a few that actually tunneled (in the two lower quadrants). For $m_{q}=0.05$ the picture is similar, even though there are more tunneling events. 


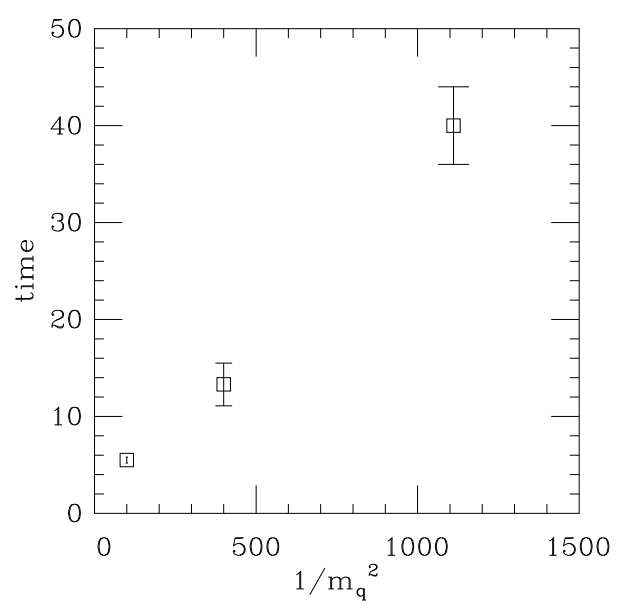

Figure 2: Monte Carlo simulation time between topology changes versus quark mass.

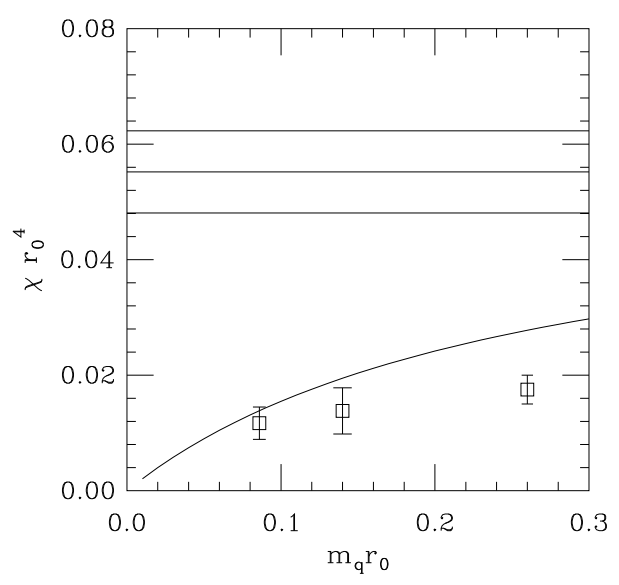

Figure 3: Topological susceptibility versus quark mass, in units of $r_{0}$. The curved line is the Dürr interpolating formula, Eq. 3.1. The three horizontal lines give the quenched value and its error.

\section{The Condensate from Eigenmode Distributions}

It was proposed more than a decade ago that the distribution of the low-lying eigenvalues of the QCD Dirac operator in a finite volume can be predicted by random matrix theory (RMT) [20, 21. 22]. Since then this hypothesis has received impressive support from lattice calculations, mainly quenched simulations [23, 24, 25, 26, 27, 28, but also some dynamical ones using staggered quarks [29, 30].

Typically, the predictions are made in the so-called epsilon regime, for which $1 / \Lambda \ll L \ll$ $1 / m_{\pi}$ with $\Lambda$ a typical hadronic scale. However, it has been found that they describe the data in a wider range. Two recent large scale studies, e.g., using the overlap operator on quenched configurations [27, 28], needed lattices with a length larger than $1.2-1.5 \mathrm{fm}$ for RMT predictions match the result of the simulation. Our dynamical lattices have a spatial extent of about $1.3 \mathrm{fm}$. As we will see, random matrix theory describes our low-lying Dirac spectra quite well. 
Our analysis is based on the distribution of the $k$-th eigenmode from RMT as presented in Ref. [31] and successfully compared to simulation results in Ref. [30]. The prediction is for the distribution of the dimensionless quantity $\zeta=\rho \lambda_{k} \Sigma V$ in each topological sector, with $\lambda_{k}$ the $k$ th eigenvalue of the Dirac operator, $\Sigma$ the chiral condensate, and $V$ the volume of the box. The quantity $\rho$ is the one-loop finite volume correction, $\rho=1+c /\left(f_{\pi} L\right)^{2}$ where $c$ is a "shape factor." These distribution are universal and depend only on the number of flavors, the topological charge and the dimensionless quantity $m_{q} \Sigma V$.

By comparing the distribution of the eigenmodes with the RMT prediction one can thus measure the chiral condensate $\Sigma$. The main advantage of this method is that it gives the zero quark mass, infinite volume condensate directly. The validity of the approach can be verified comparing the shape of the distribution for the various modes and topological sectors. The main uncertainty comes from a too small volume which causes deviations in the shape, particularly for the higher modes.

In Fig. 4 we show the distribution of the two lowest eigenmodes of the overlap operator (scaled by $\Sigma V$ ) measured on the $v=0$ and $v= \pm 1$ parts of the $a m_{q}=0.03$ and $a m_{q}=0.05$ ensembles. We fit the RMT prediction from Ref. [31] to these distributions. The prediction agrees overall well with the measured distribution given the low statistics. However, the distribution of the lowest mode in the $v=0$ sector seems to have a tail at larger $\lambda \Sigma V$ that does not match the prediction. This could be an effect of the small volume. We also show the prediction for the distribution of the third mode from our fitted values of $\Sigma V$ in the third column of Fig. 4 . The RMT curve and the data, again, agree quite well. However for the $|v|=1$ sector, the curve seems to be on the right of the data. This is probably a sign of the breakdown of RMT for eigenvalues larger than the Thouless energy [32, 25].

\begin{tabular}{c|c}
$a m_{q}$ & $\rho \Sigma r_{0}^{3}$ \\
\hline 0.05 & $0.40(2)$ \\
0.03 & $0.44(2)$ \\
0.01 & $0.38(2)$ \\
\hline
\end{tabular}

Table 1: Condensate versus quark mass.

A combined fit to $v=0,1, n=1,2$ at each $m_{q}$ (four distributions fit simultaneously) gives the results shown in Table 1. In our small volumes, and using the physical value for $f_{\pi}(93 \mathrm{MeV}), \rho \sim 1.4$, which is uncomfortably large. Dividing it out boldly gives $\Sigma \sim(280 \mathrm{MeV})^{3}$.

After Ref. [6] appeared, we performed some simulations at lower quark mass, $a m_{q}=0.01$. We restricted the topological sector to $v=0$ by forbidding refractions. It is unknown whether a particular topological sector is simply connected or not (in the latter case, forbidding topology changes might make the simulation non-ergodic). We ran two separate molecular dynamics streams to look for any obvious discrepancies. We did not see any: In the two streams, the plaquette and string tension parameters were consistent within statistical uncertainties; nothing looks unusual. We also did some running at $a m=0.005$ (or a $5 \mathrm{MeV}$ quark mass), though not with enough statistics to fit the condensate. In both cases the code ran stably and quietly.

Fig. 5 shows the distributions and fit to the condensate from this data set.

\section{Conclusions}

Simulations with dynamical overlap fermions are poised to begin producing physics results. We are presently simulating on larger volumes in order to make a more reliable calculation of the condensate. We also continue to groom our algorithms. We believe that there are many more tricks 

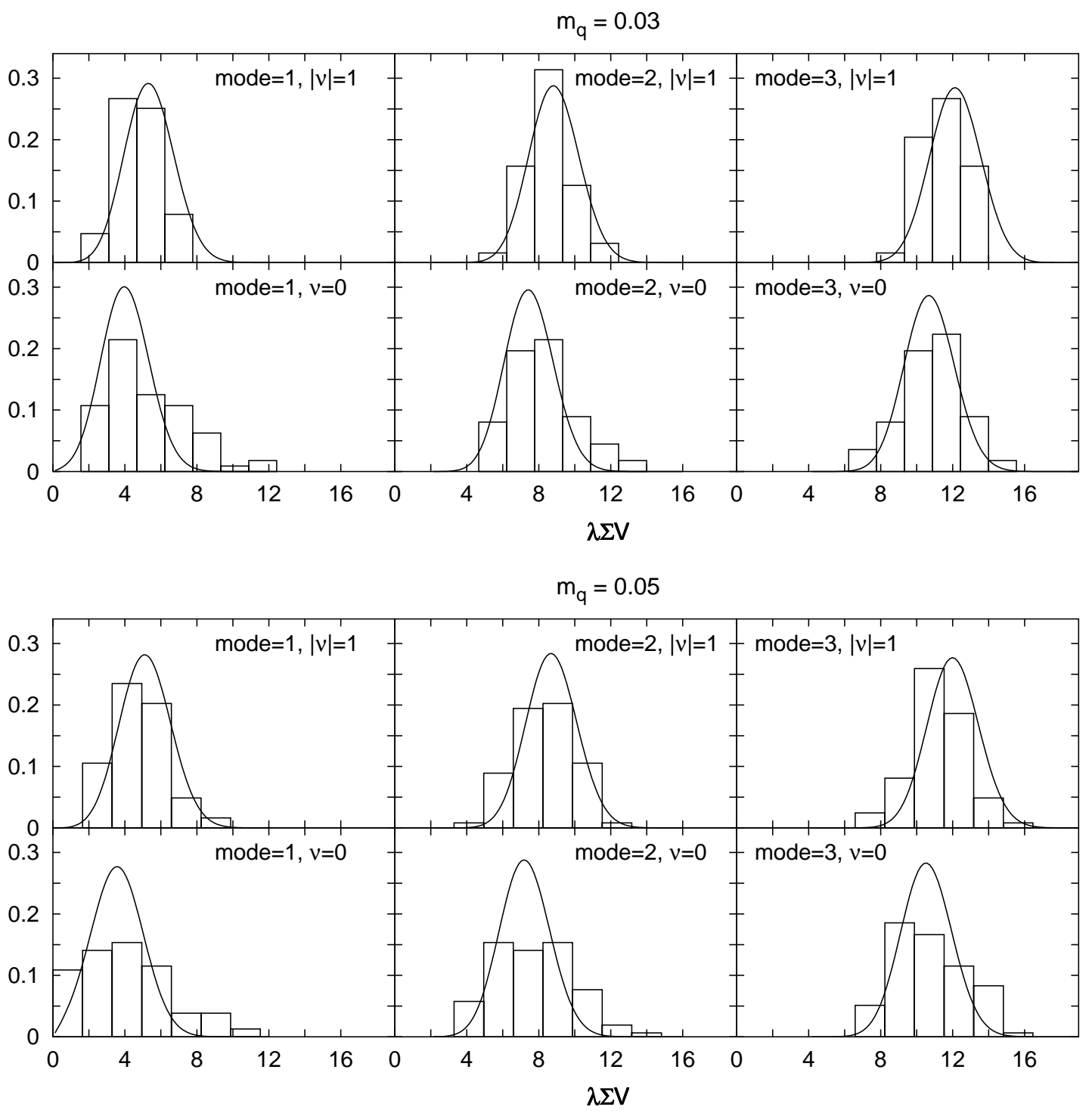

Figure 4: Distribution of the lowest two eigenmodes of the Dirac operator for our ensemble for $v=0, \pm 1$. The lines are fits of the random matrix theory prediction to the data for the two lowest modes. The lines for the third mode are predictions.

to be found and encourage others to work on dynamical simulations with overlap fermions. The physics payoffs are potentially very high.

This work was supported by the US Department of Energy.

\section{References}

[1] P. H. Ginsparg and K. G. Wilson, A remnant of chiral symmetry on the lattice, Phys. Rev. D25 (1982) 2649. 
[2] H. Neuberger, Exactly massless quarks on the lattice, Phys. Lett. B417 (1998) 141-144, [hep-lat/9707022].

[3] H. Neuberger, A practical implementation of the overlap-dirac operator, Phys. Rev. Lett. 81 (1998) 4060-4062, hep-lat/9806025.

[4] Z. Fodor, S. D. Katz, and K. K. Szabo, Dynamical overlap fermions, results with hybrid monte-carlo algorithm, JHEP 08 (2004) 003, [hep-lat/0311010].

[5] T. DeGrand and S. Schaefer, Physics issues in simulations with dynamical overlap fermions, Phys. Rev. D71 (2005) 034507, [hep-lat/0412005].

[6] T. DeGrand and S. Schaefer, Chiral properties of two-flavor qcd in small volume and at large lattice spacing, hep-lat/0506021.

[7] MILC Collaboration, T. DeGrand, Tests of hypercubic fermion actions, Phys. Rev. D58 (1998) 094503, [hep-lat/9802012].

[8] T. DeGrand, A. Hasenfratz, and T. G. Kovacs, Instantons and exceptional configurations with the clover action, Nucl. Phys. B547 (1999) 259-280, hep-lat/9810061.

[9] J. F. Lagae and D. K. Sinclair, Improved staggered quark actions with reduced flavour symmetry violations for lattice qcd, Phys. Rev. D59 (1999) 014511, hep-lat/9806014].

[10] MILC Collaboration, K. Orginos, D. Toussaint, and R. L. Sugar, Variants of fattening and flavor symmetry restoration, Phys. Rev. D60 (1999) 054503, hep-lat/9903032.

[11] C. Morningstar and M. J. Peardon, Analytic smearing of su(3) link variables in lattice qcd, Phys. Rev. D69 (2004) 054501, hep-lat/0311018.

[12] J. C. Sexton and D. H. Weingarten, Hamiltonian evolution for the hybrid monte carlo algorithm, Nucl. Phys. B380 (1992) 665-678.

[13] G. Golub and C. F. Van Loan, Matrix Computations. The Johns Hopkins University Press, Baltimore, 3rd ed., 1996.

[14] M. Hasenbusch, Speeding up the hybrid-monte-carlo algorithm for dynamical fermions, Phys. Lett. B519 (2001) 177-182, [hep-lat/0107019].

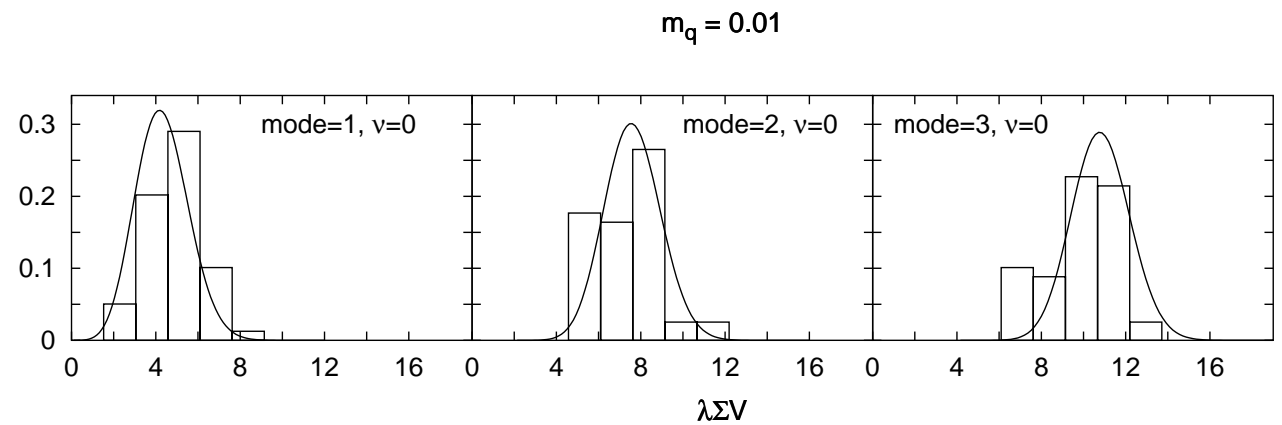

Figure 5: Distribution of the lowest two eigenmodes of the Dirac operator for our ensemble for $v=0$ at $a m_{q}=0.01$. The lines are fits of the random matrix theory prediction to the data for the two lowest modes. The lines for the third mode are predictions. 
[15] M. Hasenbusch and K. Jansen, Speeding up lattice qcd simulations with clover-improved wilson fermions, Nucl. Phys. B659 (2003) 299-320, [hep-lat/0211042.

[16] S. Durr, Topological susceptibility in full qcd: Lattice results versus the prediction from the qcd partition function with granularity, Nucl. Phys. B611 (2001) 281-310, hep-lat/0103011.

[17] Z. Fodor, S. D. Katz, and K. K. Szabo, Dynamical overlap fermions, results with hmc algorithm, hep-lat/0409070.

[18] A. Hasenfratz, Topological susceptibility on dynamical staggered fermion configurations, Phys. Rev. D64 (2001) 074503, hep-lat/0104015].

[19] UKQCD Collaboration, C. R. Allton et al., Improved wilson qcd simulations with light quark masses, Phys. Rev. D70 (2004) 014501, [hep-lat/0403007].

[20] E. V. Shuryak and J. J. M. Verbaarschot, Random matrix theory and spectral sum rules for the dirac operator in qcd, Nucl. Phys. A560 (1993) 306-320, [hep-th/9212088.

[21] J. J. M. Verbaarschot and I. Zahed, Spectral density of the qcd dirac operator near zero virtuality, Phys. Rev. Lett. 70 (1993) 3852-3855, [hep-th/9303012].

[22] J. J. M. Verbaarschot, The spectrum of the qcd dirac operator and chiral random matrix theory: The threefold way, Phys. Rev. Lett. 72 (1994) 2531-2533, [hep-th/9401059.

[23] M. E. Berbenni-Bitsch, S. Meyer, A. Schafer, J. J. M. Verbaarschot, and T. Wettig, Microscopic universality in the spectrum of the lattice dirac operator, Phys. Rev. Lett. 80 (1998) 1146-1149, hep-lat/9704018.

[24] P. H. Damgaard, U. M. Heller, and A. Krasnitz, Microscopic spectral density of the dirac operator in quenched qcd, Phys. Lett. B445 (1999) 366-370, hep-lat/9810060.

[25] M. Gockeler, H. Hehl, P. E. L. Rakow, A. Schafer, and T. Wettig, Small eigenvalues of the su(3) dirac operator on the lattice and in random matrix theory, Phys. Rev. D59 (1999) 094503, [hep-lat/9811018].

[26] R. G. Edwards, U. M. Heller, J. E. Kiskis, and R. Narayanan, Quark spectra, topology and random matrix theory, Phys. Rev. Lett. 82 (1999) 4188-4191, [hep-th/9902117].

[27] W. Bietenholz, K. Jansen, and S. Shcheredin, Spectral properties of the overlap dirac operator in qcd, JHEP 07 (2003) 033, hep-lat/0306022.

[28] L. Giusti, M. Luscher, P. Weisz, and H. Wittig, Lattice qcd in the epsilon-regime and random matrix theory, JHEP 11 (2003) 023, [hep-lat/0309189].

[29] M. E. Berbenni-Bitsch, S. Meyer, and T. Wettig, Microscopic universality with dynamical fermions, Phys. Rev. D58 (1998) 071502, [hep-lat/9804030].

[30] P. H. Damgaard, U. M. Heller, R. Niclasen, and K. Rummukainen, Eigenvalue distributions of the qcd dirac operator, Phys. Lett. B495 (2000) 263-270, [hep-lat/0007041].

[31] P. H. Damgaard and S. M. Nishigaki, Distribution of the $k$-th smallest dirac operator eigenvalue, Phys. Rev. D63 (2001) 045012, [hep-th/000611]].

[32] J. C. Osborn and J. J. M. Verbaarschot, Thouless energy and correlations of QCD dirac eigenvalues, Phys. Rev. Lett. 81 (1998) 268-271, hep-ph/9807490. 